\title{
Time Interference Alignment via Delay Offset for Long Delay Networks
}

\author{
Francisco Lázaro Blasco, Francesco Rossetto, Gerhard Bauch
}

\begin{abstract}
The potential of Time Interference Alignment is investigated in this work, with particular reference to the attainable degrees of freedom. The $K$-user interference channel is considered, in which transmitters and receivers are placed randomly in a Euclidean space. A model for long delay networks is introduced and the degrees of freedom for different cases (with and without transmitter delay coordination) are evaluated. It is shown how time interference alignment can provide more degrees of freedom than TDMA when the transmitters jointly coordinate their transmission delay and the number of pairs is $K \geq 5$. Closed form expressions are derived for several cases of interest which provide insight and useful predictions. This work is concluded with an investigation of the achievable degrees of freedom for multi-satellite networks, where it is shown that the results obtained under several assumptions do predict accurately the degrees of freedom in a real setting.
\end{abstract}

\section{INTRODUCTION}

The significant capacity improvement possible through Interference Alignment (IA) has attracted growing interest. The possibility to attain the degrees of freedom (dof) ${ }^{1}$ of some important network topologies has theoretically supported the relevance of this technique [1]-[3]. The potential gains have been verified in practice by some testbeds [4], [5]. In certain

This work was presented in part at IEEE GLOBECOM 2011. Francisco Lázaro Blasco is with the Institute of Communications and Navigation, DLR (German Aerospace Center), Wessling, Germany 82234. Francesco Rossetto was with DLR, he is now with Rohde \& Schwarz, Mühldorferstraße 15, 81671 Munich, Germany. Gerhard Bauch was with the Department for Communications Engineering, Universität der Bundeswehr, Munich, Germany, he is now with the Technische Universität Hamburg-Harburg, Germany. Email: Francisco.LazaroBlasco@dlr.de, Francesco.Rossetto@ieee.org, bauch@tuhh. de.

This work has been accepted for publication in IEEE Transactions on Communications.

(c) 2013 IEEE. Personal use of this material is permitted. Permission from IEEE must be obtained for all other uses, in any current or future media, including reprinting/republishing this material for advertising or promotional purposes, creating new collective works, for resale or redistribution to servers or lists, or reuse of any copyrighted component of this work in other works

${ }^{1}$ The sum dof for a network and a certain transmission scheme are defined as:

$$
d o f=\lim _{P \rightarrow+\infty} \frac{C(P)}{\log (P)},
$$

where $P$ is the transmit power and $C(P)$ is the sum rate of the network. settings (as in the $K$-user interference channel) the capacity can even grow linearly with the number of terminals [1].

All types of IA require that the signal lives in a space with multiple dimensions. The core idea that underpins IA is to divide this space into a desired subspace (where the intended signal should lie) and confine all interference into an interference subspace. Hence, the desired subspace is interference free and thus the capacity can grow as the Signal to Noise Ratio (SNR) increases. For instance, in MIMO based IA, each signal is generated and received by multiple antennas and hence it is a vector. The employment of multiple antennas in conjunction with IA has basked a high level of attention [1], [4]-[12] but it is by no means the only way to create signals in multiple dimensions. Another possibility is the usage of different signal levels [9] or to exploit the different propagation delays between terminals [13]. Our work focuses on this last approach.

Interference alignment in the time domain, time IA for short, works by letting transmitters coordinate the start time of their transmissions so that the desired signal is received interference free at the receivers. Time IA is of particular interest for networks with long delays, such as satellite and underwater networks. In this setting, one argument in favour of Time IA is that it requires only knowledge of the transmission delays, which can be easily estimated, for example by means of a GPS receiver. Other techniques such as MIMO IA require knowledge of the complex gain of the fading channel, which is arguably more difficult to estimate, especially with mobile terminals. The idea of arranging the transmission delays in order to minimize interference was present in one of the first works on interference alignment [13] and it has received some attention in the past [1], [13]-[18]. The reference scenario is the $K$-user interference channel (see Fig. 1), where $K$ transmitters communicate with a dedicated receiver (one per sender) and all nodes are assumed to have just one antenna. In [1], [13] it was shown that there exist propagation delays that enable every user to transmit $50 \%$ of the time interference free. The feasibility of perfect IA in a Euclidean space was studied in [14]-[16]. In [14], [15] it is assumed that the transmitters are not allowed to delay their transmission and that the time allocation is the one 
shown in Fig. 1. Under these assumptions it is shown that in an $N$ dimensional Euclidean space it is possible to place $N+1$ pairs that can transmit interference free $50 \%$ of the time, therefore it is possible to attain $(N+1) / 2$ dof. In [16] a more general system model is introduced based on polynomials that describe cyclic permutations. Under the assumption that the delays between transmitters and receivers are positive integer multiples of one time slot, the conditions necessary to achieve perfect IA are derived. Contrary to this stream of research that looks for specific delay configurations that enable high capacity, [18] does not make any assumption on the values of the delays and instead attempts to organize the transmissions so as to maximise the attainable degrees of freedom with the given propagation delays. In [1], [2], [9], [13] a time slotted scheme is considered in which all transmitters transmit in even time slots and remain silent in odd time slots, hence, the transmission pattern has a period of 2 time-slots and is the same for all transmitters. In [18] different transmitters have generally different transmission patterns and the period of the transmission pattern is longer than 2 time slots. The main finding in [18] is that the dof of the network can grow linearly with the number of pairs, but only if the bandwidth of the network grows also with the number of users. This last phenomenon is referred to as bandwidth scaling.

In the present work, contrary to [1], [2], [9], [13] and together with [18], the propagation delays are random variables that cannot be controlled by the network. By other words, a special arrangement of the terminal positions is neither sought nor assumed. Instead, we find more practically relevant the question of better characterising the dof that are achieved when the transmitters arrange their delays so as to maximise the interference free time. In our analysis we focus on the evaluation of the dof under the assumption that the bandwidth of the network stays constant (no bandwidth scaling).

Our main contribution is the analysis of three approaches to time IA: no coordination (NoC), altruistic time IA (AIA) and joint maximization (JMax) (Section III, IV-A and IV-B, respectively). The first system offers a performance benchmark, leads to the derivation of several closed form expressions and provides also useful analytical tools for the other two approaches. The second scheme employs a simple alignment algorithm, which reduces the interference at undesired receivers and leads also to the derivation of analytical expressions. The last approach is based on the analysis of the two other schemes: it numerically maximises the sum dof and shows the achievable gains in terms of sum dof for Time IA. Based on the presented results, we conjecture that the sum dof grows without bound (even without bandwidth scaling), although sublinearly with the network size. Compared to previous work, the transmission pattern employed by the transmitters is more general. Moreover, helpful formulae are developed which allow to make predictions also for some real-world scenarios. Finally the applicability of the schemes is demonstrated in the context of a multi-satellite network.

The paper is structured as follows. Section II introduces the system model considered in our work. Section III presents results for the case in which the transmitters do not coordinate. Results for coordinated transmitters follow in Section IV. Numerical results for a real satellite application scenario are presented in Section V. Finally, the conclusions are drawn in Section VI.

\section{SySTEM MOdEL}

Matrices are denoted by capital letters and $m_{i, j}$ stands for the element at the $i$-th row and $j$-th column of a matrix $M$. Furthermore $M \bmod p$ represents the elementwise modulo $p$ operator of matrix $M$, by other words the remainder of the elements in the matrix $M$ with respect to a real valued divisor $p$. The rectangle function or unit pulse centered around 0 and of length 1 is expressed as $\operatorname{rect}(x)$ and $\delta(x)$ denotes the Dirac's delta. $\mathbb{R}^{N}$ stands for the $N$-dimensional Euclidean space, where $N$ is generic.

The $K$-user interference channel is considered [1], [9]. In the $K$-user interference channel $K$ transmitters communicate with $K$ receivers and, since the network is fully connected, the transmission from each of the $K$ transmitters is received at all the $K$ receivers. An example for $K=3$ is shown in Fig. 1. We shall assume that transmitter 1 communicates with receiver 1 , transmitter 2 with receiver 2 and so on. Let us consider the general case in which transmitters and receivers are placed in $\mathbb{R}^{N}$ and the delay between two nodes is proportional to their Euclidean distance. For simplicity we assume that the position of all nodes is fixed. The propagation delay among all the nodes in the channel can be expressed in a $(K \times K)$ matrix $A$, where $a_{i, j} \in \mathbb{R}^{+}$is the propagation delay between transmitter $j$ and receiver $i$. Note that, since the positions of the nodes are constant, the delays among the nodes are fixed. Time is divided into slots of length $T$. Transmitters are allowed to transmit only for a time $\rho T$ in every slot, $\rho \leq \frac{1}{2}$ and the ratio of the transmit duration over the length of a time slot, $\rho$, will be called duty cycle. This framework is more general than the cases considered in literature up to now [1], [14], [15], but it also includes the canonical example of interference alignment by means of delay offsets, where the transmitters are allowed to transmit for a fraction $\rho=\frac{1}{2}$ of the time. For simplicity, all transmitters send with the same duty cycle $\rho_{i}=\rho$.

Given the fact that the time allocation scheme is 

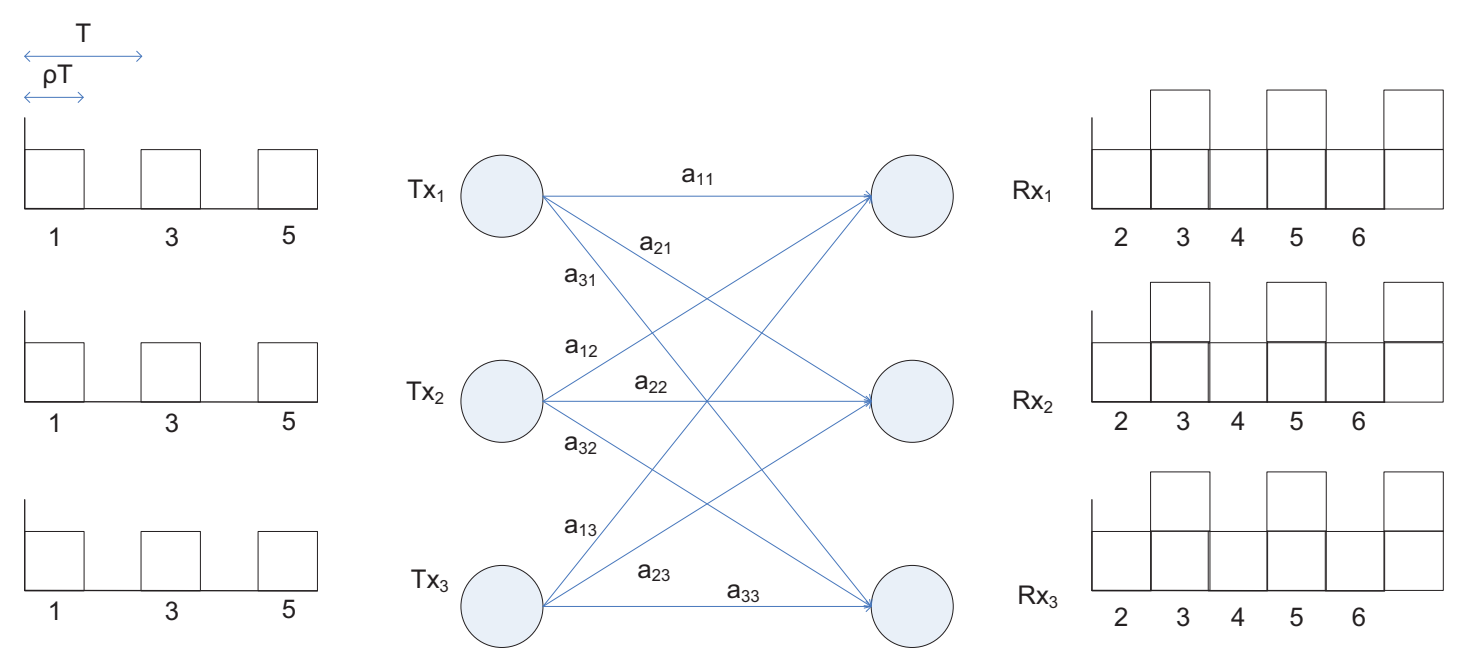

Fig. 1: The 3-user interference channel. Note that perfect IA is attained.

periodic with period $T$, it is useful to define:

$$
B=\frac{A \bmod T}{T} \text {. }
$$

$B$ is a $(K \times K)$ matrix whose elements $b_{i, j}$ take values in $[0,1)$. We refer to $B$ as normalized propagation matrix. Additionally, transmitters are allowed to delay the start of their transmission. We denote by $\Delta_{i}$ the initial transmission delay of transmitter $i$. Let us define a new matrix,

$$
D=\frac{(A+\hat{\Delta}) \bmod T}{T},
$$

which is a $(K \times K)$ matrix whose elements $d_{i, j}$ also have range $[0,1) . \hat{\Delta}$ is a $(K \times K)$ matrix whose elements, $\hat{\Delta}_{i, j}$, correspond to the initial transmit delay $\Delta_{j}$ of transmitter $j, \hat{\Delta}_{i, j}=\Delta_{j}, \forall i$.

\section{ANALYSIS FOR NON-COORDINATED TRANSMITTERS}

The first setting to be studied assumes no coordination among the transmitters, so that the initial transmission delay of each transmitter is random. We use the shorthand NoC to refer to this method. This setting represents first a benchmark for the true IA schemes, but it is also amenable to analysis and it is therefore possible to derive many closed form expressions, which constitute the basis of the investigation in those cases when the transmitters do coordinate with each other.

Transmitters and receivers are assumed to be randomly placed in a "sufficiently large" section of $\mathbb{R}^{N},{ }^{2}$ so that the elements in $A, a_{i, j}$ take values in $[0, L]$, with

\footnotetext{
${ }^{2}$ Section V will show an example of what "sufficiently large" means in a real satellite scenario for different values of $T$.
}

$L>T$. Under this assumption, we can infer that the elements $B, b_{i, j}$ are independent and uniformly distributed between 0 and 1 . When no coordination is assumed, the transmitter delays $\Delta_{i}$ are independent and uniformly distributed between 0 and $T$. Hence, the elements in $D$ are independent and uniformly distributed between 0 and 1 .

Without loss of generality we shall focus on the dof achieved by pair $i$. Let us denote by $\alpha_{i, K}$ the dof attained by pair $i$ when $K$ pairs are present. The main metric of interest is the probability density function (pdf) of $\alpha_{i, K}$, $f\left(\alpha_{i, K}\right)$. In the following we will compute $f\left(\alpha_{i, K}\right)$ in a recursive manner. As a starting point we assume that, when $K$ pairs are present, the dof achieved by pair $i$ are known and are equal to $\alpha_{i, K} \leq \rho$. We will assume a new pair joins the network, and derive the pdf $f\left(\alpha_{i, K+1}\right)$ starting from $f\left(\alpha_{i, K}\right)$.

Every period $T$, receiver $i$ sees the signal from transmitter $i$ interference free for a contiguous time $\alpha_{i, K} T$. Let us assume that the interference free signal at receiver $i$ starts at time $\tau T$. Transmitter $K+1$ transmits for contiguous time $\rho T$ every period $T$. For simplicity the auxiliary variable $\eta$ is introduced and represents the difference between the starting time of the interference from transmitter $K+1, d_{i, K+1}$ and $\tau$ modulo $1, \eta=\left(d_{i, K+1}-\tau\right) \quad \bmod 1$.

In the first case the interference introduced by the $K+$ 1-th transmitter does not overlap with the interference free signal from transmitter $i$ at receiver $i$. This case is depicted in Fig. 2. More concretely the two extreme cases are shown, $\eta=\alpha_{i, K}$ and $\eta=1-\rho$. The probability of this case is:

$$
\begin{aligned}
\operatorname{Pr}\left(\alpha_{i, K+1}\right. & \left.=\alpha_{i, K} \mid \alpha_{i, K}\right)=\operatorname{Pr}\left(\alpha_{i, K}<\eta<(1-\rho)\right) \\
& =1-\rho-\alpha_{i, K} .
\end{aligned}
$$




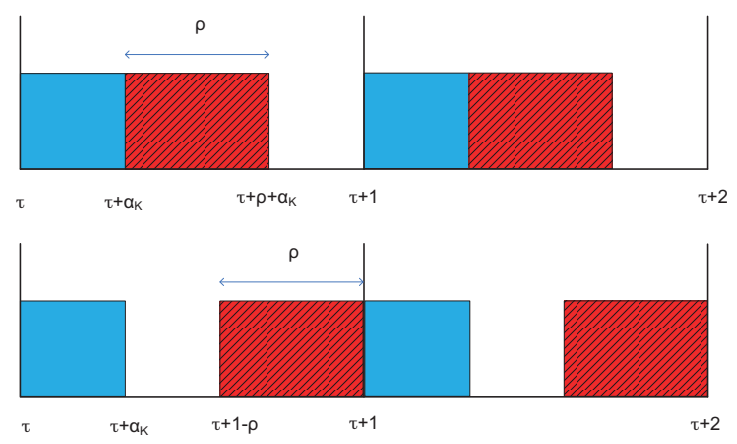

Fig. 2: Snapshot from time $\tau$ to $\tau+2$ at receiver $i$ when the $K+1$-th transmitter does not overlap with the interference free signal from transmitter $i$. Time has been normalized to the time period $T$. The solid block represents the useful signal whereas the striped block represents the interference. The upper part represents $\eta=\alpha_{K}$ and the lower part $\eta=1-\rho$.

The second possibility corresponds to the case in which the signal from transmitter $K+1$ completely overlaps with the interference free signal at receiver $i$, and therefore $\alpha_{i, K+1}=0$. The probability of this event is :

$$
\begin{aligned}
\operatorname{Pr}\left(\alpha_{i, K+1}=0 \mid \alpha_{i, K}\right) & =\operatorname{Pr}\left(1-\left(\rho-\alpha_{i, K}\right)<\eta<1\right) \\
& =\rho-\alpha_{i, K} .
\end{aligned}
$$

The third case corresponds to $\alpha_{i, K+1}$ taking values between 0 and $\alpha_{i, K}$ :

$$
\begin{aligned}
\operatorname{Pr}\left(\alpha_{i, K+1} \mid \alpha_{i, K}\right) & =\operatorname{Pr}\left(\alpha_{i, K+1} \leq \alpha \mid \alpha_{i, K}\right) \\
& =1-\operatorname{Pr}\left(\alpha_{i, K+1}>\alpha \mid \alpha_{i, K}\right) \\
& =1-\operatorname{Pr}\left(\alpha<\eta<1+\alpha_{K}-\alpha-\rho\right) \\
& =\rho-\alpha_{i, K}+2 \alpha
\end{aligned}
$$

By taking the derivative of (6), and taking into consideration also the probability mass points (4) and (5), it is possible to obtain the pdf of $\alpha_{i, K+1}$ conditioned to $\alpha_{i, K}$ :

$$
\begin{aligned}
f\left(\alpha_{i, K+1} \mid \alpha_{i, K}\right) & =\frac{\partial \mathbf{F}\left(\alpha_{i, K+1} \mid \alpha_{i, K}\right)}{\partial \alpha_{i, K+1}} \\
& =2 \operatorname{rect}\left(\frac{\alpha_{i, K+1}-\frac{\alpha_{i, K}}{2}}{\alpha_{i, K}}\right) \\
& +\left(\rho-\alpha_{i, K}\right) \delta\left(\alpha_{i, K+1}\right) \\
& +\left(1-\rho-\alpha_{i, K}\right) \delta\left(\alpha_{i, K+1}-\alpha_{i, K}\right),
\end{aligned}
$$

where $\mathbf{F}\left(\alpha_{i, K+1} \mid \alpha_{i, K}\right)$ is the cumulative distribution function of $\alpha_{i, K+1}$ conditioned to $\alpha_{i, K}$, which is obtained from (4), (5) and (6). If the pdf of $\alpha_{i, K}$ is known, the pdf of $\alpha_{i, K+1}$ can be calculated as:

$$
f\left(\alpha_{i, K+1}\right)=\int_{-\infty}^{\infty} f\left(\alpha_{i, K+1} \mid \alpha_{i, K}\right) f\left(\alpha_{i, K}\right) \mathrm{d} \alpha_{i, K} .
$$

In this way the pdf of $\alpha_{i, K}$ is computed recursively. In the following the index $i$ is dropped for the sake of notational simplicity. Hence, $\alpha_{K}$ denotes the dof achieved by a generic pair of users when a total of $K$ pairs is present in the network. The starting point is the case with only one pair. Since there are no interferers $\alpha_{1}=\rho$ with probability 1 :

$$
f\left(\alpha_{1}\right)=\delta\left(\alpha_{1}-\rho\right) .
$$

Applying (8), the expression for $f\left(\alpha_{2}\right)$ is obtained:

$f\left(\alpha_{2}\right)=2 \operatorname{rect}\left(\frac{\alpha_{2}-\frac{\rho}{2}}{\rho}\right)+(1-2 \rho) \delta\left(\alpha_{2}-\rho\right)$.

For $K=3$ the following expression is obtained:

$$
\begin{aligned}
f\left(\alpha_{3}\right) & =\left(4-2 \rho-6 \alpha_{3}\right) \operatorname{rect}\left(\frac{\alpha_{3}-\frac{\rho}{2}}{\rho}\right) \\
& +\rho^{2} \delta\left(\alpha_{3}\right)+\left(1-4 \rho+4 \rho^{2}\right) \delta\left(\alpha_{3}-\rho\right) .
\end{aligned}
$$

And for $K=4$ we have:

$$
\begin{aligned}
f\left(\alpha_{4}\right) & =\left(\rho^{3}+(1-\rho) \rho^{2}+2(1-\rho) \rho^{2}\right) \delta\left(\alpha_{4}\right) \\
& +(1-2 \rho)^{3} \delta\left(\alpha_{4}-\rho\right)+\left(2(1-2 \rho)^{2}\right. \\
& +\left(4-2 \rho-6 \alpha_{4}\right)\left(1-\rho-\alpha_{4}\right) \\
& \left.+2\left(4-3 \alpha_{4}-5 \rho\right)\left(\rho-\alpha_{4}\right)\right) \operatorname{rect}\left(\frac{\alpha_{4}-\frac{\rho}{2}}{\rho}\right) .
\end{aligned}
$$

The expression for $f\left(\alpha_{K}\right)$ for $K>4$ is calculated in the same way simply by applying the recursive iteration in (8). It is clear that the computation of the pdf of $\alpha_{K}$ poses no challenge in principle but it becomes rather tedious, especially for large $K$.

Applying (8), a simple recursive expression for $\mathbf{E}\left[\alpha_{K+1}\right]$ is obtained:

$$
\begin{aligned}
& \mathbf{E}\left[\alpha_{K+1}\right]=\int_{-\infty}^{\infty} \alpha_{K+1} f\left(\alpha_{K+1}\right) \mathrm{d} \alpha_{K+1} \\
& =\int_{-\infty}^{\infty} \alpha_{K+1}\left(\int_{-\infty}^{\infty} f\left(\alpha_{K+1} \mid \alpha_{K}\right) f\left(\alpha_{K}\right) \mathrm{d} \alpha_{K}\right) \mathrm{d} \alpha_{K+1} \\
& =\int_{-\infty}^{\infty}\left(\int_{-\infty}^{\infty} \alpha_{K+1} f\left(\alpha_{K+1} \mid \alpha_{K}\right) \mathrm{d} \alpha_{K+1}\right) f\left(\alpha_{K}\right) \mathrm{d} \alpha_{K} \\
& =\int_{-\infty}^{\infty}\left(\int _ { - \infty } ^ { \infty } \alpha _ { K + 1 } \left(2 \operatorname{rect}\left(\frac{\alpha_{K+1}-\frac{\alpha_{K}}{2}}{\alpha_{K}}\right)+\left(\rho-\alpha_{K}\right) \delta\left(\alpha_{K+1}\right)\right.\right. \\
& \left.\left.+\left(1-\rho-\alpha_{K}\right) \delta\left(\alpha_{K+1}-\alpha_{K},\right)\right) \mathrm{d} \alpha_{K+1}\right) f\left(\alpha_{K}\right) \mathrm{d} \alpha_{K} \\
& =\int_{-\infty}^{\infty}\left(\int_{0}^{\alpha_{K}} 2 \alpha_{K+1} \mathrm{~d} \alpha_{K+1}+0+\left(1-\rho-\alpha_{K}\right) \alpha_{K}\right) f\left(\alpha_{K}\right) \mathrm{d} \alpha_{K} \\
& =\int_{-\infty}^{\infty}(1-\rho) \alpha_{K} f\left(\alpha_{K}\right) \mathrm{d} \alpha_{K} \\
& =(1-\rho) \int_{-\infty}^{\infty} \alpha_{K} f\left(\alpha_{K}\right) \mathrm{d} \alpha_{K} \\
& =(1-\rho) \mathbf{E}\left[\alpha_{K}\right],
\end{aligned}
$$

which can be written in closed form taking into account the trivial case $\mathbf{E}\left[\alpha_{1}\right]=\rho$ :

$$
\mathbf{E}\left[\alpha_{K}\right]=\rho(1-\rho)^{K-1} .
$$

It is easy now to prove that the value of $\rho$ which maximizes $\mathbf{E}\left[\alpha_{K}\right]$ is:

$$
\rho_{K}^{N o C}=\frac{1}{K} .
$$

So far we have characterized the $f\left(\alpha_{K}\right)$, the pdf of the dof for one pair when a total of $K$ pairs are present. Let us denote as $\phi_{K}=\sum_{i} \alpha_{i, K}$, the sum dof achieved by the $K$ pairs. We recall that the dof of achieved by each pair corresponds to portion of time in which the receiver sees the signal from its transmitter interference free. The pdf of the sum dof for $K$ users, $f\left(\phi_{K}\right)$, is simply the 
convolution of $K$ single user pdfs. In Appendix A we provide the expression of $f\left(\phi_{3}\right)$ as an example. Using (14) it is possible to provide a simple expression for $\mathbf{E}\left[\phi_{K}\right]$ :

$\mathbf{E}\left[\phi_{K}\right]=\mathbf{E}\left[\sum_{i=1}^{K} \alpha_{i, K}\right]=K \mathbf{E}\left[\alpha_{K}\right]=K \rho(1-\rho)^{K-1}$.

Writing $\phi_{K}$ as a function of $\phi_{K-1}$ gives us also some insight into how the sum degrees of freedom evolve as a function of $K$ :

$$
\mathbf{E}\left[\phi_{K}\right]=\frac{K}{K-1}(1-\rho) \mathbf{E}\left[\phi_{K-1}\right] .
$$

By means of (17) it is also possible to compute the mean sum dof when the number of pairs, $K$, goes to infinity and the optimal duty cycle $\rho_{K}^{N o C}$ is used, $\phi_{K}^{N o C}=\mathbf{E}\left[\phi_{K}\left(\rho_{K}^{N o C}\right)\right]$ :

$$
\begin{aligned}
\lim _{k \rightarrow \infty} \phi_{K}^{N o C} & =\lim _{K \rightarrow \infty} K \rho_{K}^{N o C}\left(1-\rho_{K}^{N o C}\right)^{K-1} \\
& =\lim _{K \rightarrow \infty}\left(1-\frac{1}{K}\right)^{K-1}=\frac{1}{e} .
\end{aligned}
$$

$\phi_{K}^{N o C}$ is monotonically decreasing with $K$ and tends to $\frac{1}{e}$ as the number of pairs, $K$, tends to infinity, as depicted in Fig. 3. It can be observed how $\phi_{K}^{N o C}$ decreases rapidly with $K$, and already for $K=5$ it is close to $\frac{1}{e}$.

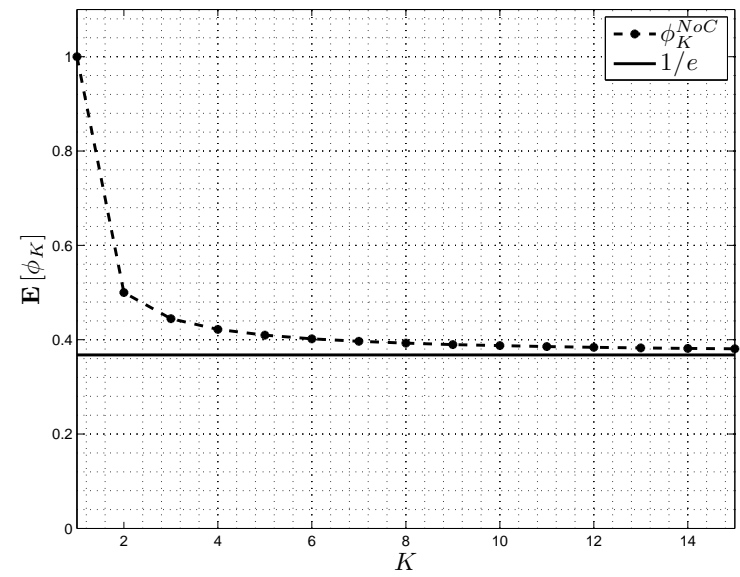

Fig. 3: $\phi_{K}^{N o C}$ vs $K$. The dashed line represents $\phi_{K}^{N o C}$. The solid line represents $\frac{1}{e}$ which is the limit of $\phi_{K}^{N o C}$ when $K$ tends to infinity.

Fig. 4 shows the CCDF of $\phi_{K}^{N o C}$ for $K \in\{2, \ldots, 5\}$. It can be seen that, as the number of users increases, the probability of $\phi_{K}^{N o C}$ taking values close to 1 decreases. Note that the CCDF of $\phi_{K}^{N o C}$ has a discontinuity at $\phi_{K}^{N o C}=1$ for $K>2$. This is because the probability of $\phi_{K}^{N o C}$ taking value 1 is not zero for $K>2$. Hence, the pdf of $\phi_{K}^{N o C}$ has a Dirac's delta at $\phi_{K}^{N o C}=1$.

As expected, when the transmitters do not coordinate the system capacity is very low, however the analysis

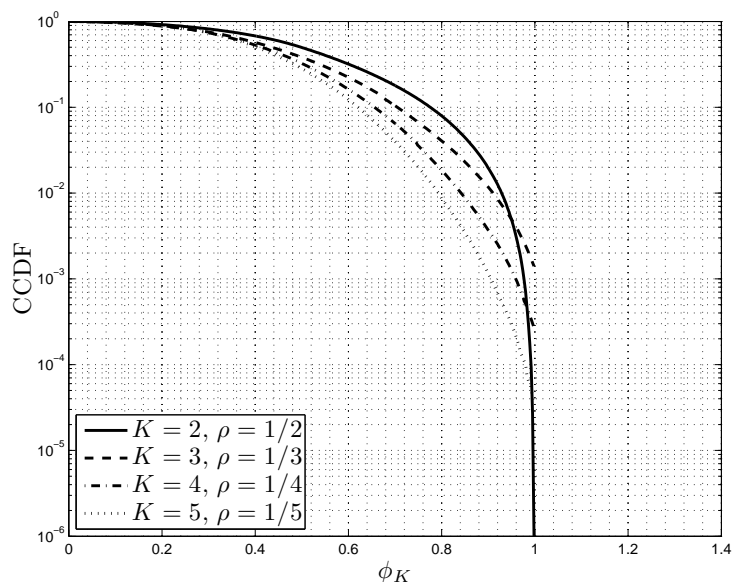

Fig. 4: CCDF of the sum dof with no coordination, $\phi_{K}^{N o C}$, for $K=2,3,4,5$.

of the uncoordinated case proves to be helpful in the investigation of coordinated transmitters, as Section IV will show.

\section{ANALYSIS FOR COORDINATED TRANSMITTERS}

In this section we assume that the transmitters have perfect knowledge of the normalized propagation delay matrix, $B$. Moreover, according to the IA paradigm, the transmitters align their transmissions so that the undesired signals at the receivers overlap as much as possible leaving the desired signal interference free. This alignment in time is achieved by letting the transmitters apply an initial delay $\Delta_{i}$. We analyze two different strategies. In the first scheme, which we call altruistic IA, transmitters align the transmissions only to reduce the interference at undesired receivers. The second strategy consists of a joint maximization of the sum dof. The first approach is studied analytically by means of the tools developed in Section III. The latter technique is built upon the numerical optimization of the sum dof of a $K$-user interference channel, and explores the capacity gains achievable by means of IA.

\section{A. Altruistic Interference Alignment}

In this scheme the transmitters adjust their transmission delays in order to reduce the interference caused at unintended receivers. We denote the scheme as altruistic time IA (AIA), since the transmitters do not consider the signal at their intended receiver in order to choose their transmission delay. This scheme is better illustrated by means of an example. Assume we have a network with 3 randomly placed pairs and let us first focus on receiver 1 . Receiver 1 has $K-1=2$ interferers, namely transmitters 2 and 3. Transmitter 2 can set its transmission delay $\Delta_{2}$ so that its interference at receiver 2 completely overlaps 
with the other interferer, transmitter 3 . In absence of coordination, receiver 1 had two interferers, and therefore the pdf of its dof was $f\left(\alpha_{3}\right)$. However, after transmitter 2 sets $\Delta_{2}$ as mentioned, it has only 1 potential ${ }^{3}$ interferer and the pdf of its dof becomes $f\left(\alpha_{2}\right)$. Furthermore, $\Delta_{1}$ can still be set to reduce the number of potential interferers at receiver 2 or 3 . Hence, the transmitters will coordinate in order to align their interference at the unintended receivers. We remark that when the transmitters optimize their transmission delays they do not take into consideration the useful signal at the desired receiver. In a network with $K$ pairs, $K-1$ transmitters can set their transmit delays in order to reduce the number of potential interferers at some receivers. Here the transmitters have different possibilities. For example, $K-1$ transmitters could coordinate to reduce the potential interferers at different receivers. Hence, $K-1$ receivers would have $K-2$ potential interferers and one receiver would have $K-1$ potential interferers. In the general setting when altruistic IA is used the sum dof can be written as:

$$
\phi_{K}=\sum_{k=1}^{K-1} u_{k} \alpha_{k+1}
$$

where $u_{k}$ represents the number of pairs with $k$ potential interferers. Note that the summation starts at $k=1$ because at most the $K-1$ interferers will align their transmission at one receiver, so that the receiver sees only one potential interferer. Since the number of pairs is $K$ the expression is subject to the constraint:

$$
\sum_{k=1}^{K-1} u_{k}=K, \quad u_{k} \in\{1,2, \ldots K\} .
$$

In order to have a pair with $i$ potential interferers, $K-1-i$ transmitters need to align their interference. Therefore, having a pair with $i$ potential interferers implies to commit $K-1-i$ free variables. Since $K-1$ free variables are available, this translates into the constraint:

$$
\sum_{i=1}^{K-1} u_{i}(K-1-i)=K-1 .
$$

Eq. (19) expresses the sum dof achieved by a system with altruistic IA as a sum of random variables. Making use of (14) and (19) the expected value of $\phi_{K}$ is:

$$
\mathbf{E}\left[\phi_{K}\right]=\sum_{k=1}^{K-1} u_{k} \rho(1-\rho)^{k},
$$

${ }^{3}$ By one potential interferer we mean that there is one transmitter which may cause interference at receiver 1 . However this transmitter need not cause interference. Under the presence of one potential interference, the dof obtained will have a probability distribution function $f\left(\alpha_{2}\right)$ as given in the previous section. subject to the constraints (20) and (21). For a given $K$, the maximisation of $\mathbf{E}\left[\phi_{K}\right]$ is performed in two steps. First, we find the optimal values of $u_{k}$ for $k \in$ $\{1, \ldots, K-1\}$ which maximize $E\left[\phi_{K}\right]$ for all values of $\rho, 0<\rho \leq \frac{1}{2}$. In Appendix B we show that $\mathbf{E}\left[\phi_{K}\right]$ is maximized for $u_{1}=1, u_{K-2}=1$ and $u_{K-1}=K-2$, so that the expression of the optimal $\mathbf{E}\left[\phi_{K}\right]$ is:

$\mathbf{E}\left[\phi_{K}\right]=\rho(1-\rho)+\rho(1-\rho)^{K-2}+(k-2) \rho(1-\rho)^{K-1}$.

Therefore, in order to maximize $\mathbf{E}\left[\phi_{K}\right]$ for all values of $\rho, K-2$ transmitters have to set their transmit delays $\Delta_{i}$ to align their signal at the same receiver, and the remaining transmitter aligns its transmission at another receiver.

For the optimal choice of $u_{k}$, it is easy to numerically compute the value $\rho_{K}^{A I A}$ which maximizes the expected sum dof when AIA is used, $\phi_{K}^{A I A}$, although no closed form formula could be found. Fig. 5 shows $\rho_{K}^{A I A}$ for $K=3,4, \ldots, 20$. We can see how $\rho_{K}^{A I A}>\rho_{K}^{N o C}$, although $\rho_{K}^{A I A}$ approaches $\rho_{K}^{N o C}$ as $K$ increases.

Fig. 6 shows $\phi_{K}^{A I A}$ for $K \in\{3, \ldots, 20\}$. This figure shows how altruistic IA provides a dof gain with respect to the uncoordinated case, $\phi_{K}^{A I A}>\phi_{K}^{N o C}$. However, this gain decreases with $K$, and when $K$ tends to infinity $\phi_{K}^{A I A}$ also tends to $\frac{1}{e}$. This outcome was verified numerically and not analytically since it is very challenging to compute in closed form $\rho_{K}^{A I A}$.

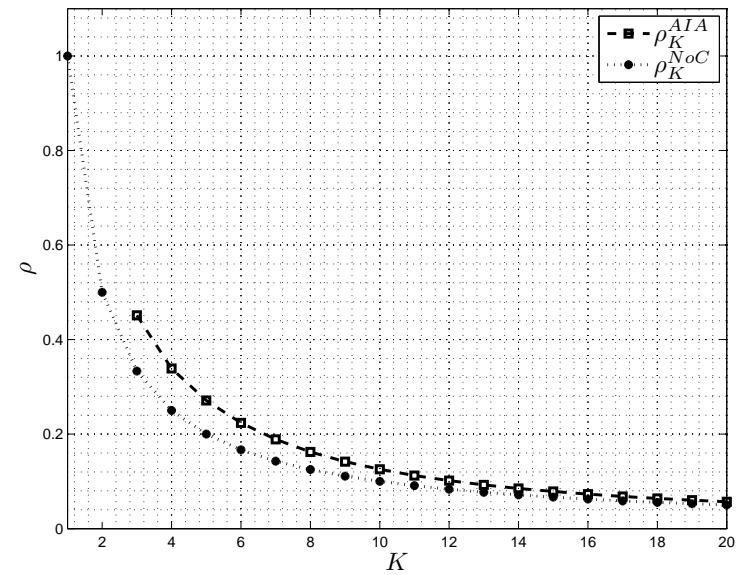

Fig. 5: The dashed line with square markers shows $\rho_{K}^{A I A}$ whereas the dotted line with round markers represents $\rho_{K}^{N o C}$.

\section{B. Joint maximization of the sum dof}

In this section a new type of coordination is introduced which aims to jointly maximize the sum dof $\phi_{K}$ and this will be referred to as JMax. Coordination has now two simultaneous goals: first, the minimization of the mutual interference at undesired receivers and secondly 


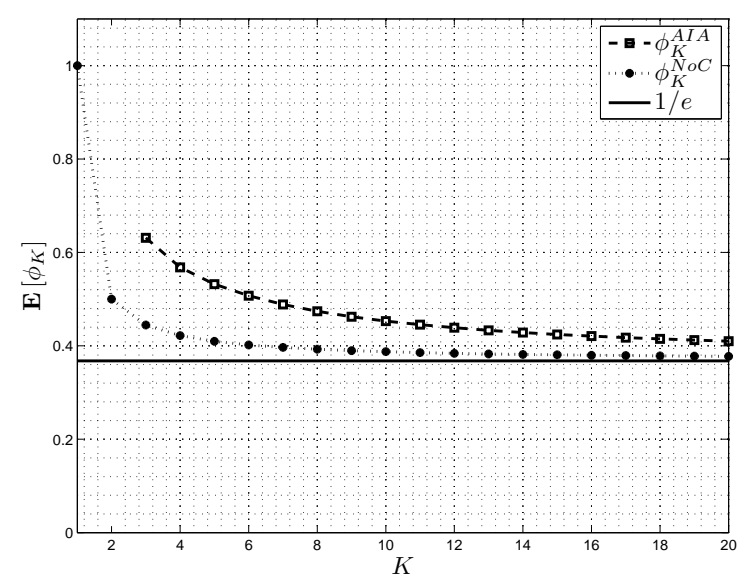

Fig. 6: Sum dof vs $K$ for the AIA and NoC schemes. The dashed line with square markers represents $\phi_{K}^{A I A}$, the dotted line with round markers represents $\phi_{K}^{N o C}$ and the solid line represents $\frac{1}{e}$.

the maximization of the useful signal at the desired receiver. This joint optimization is non trivial generally speaking and it has been performed numerically in a centralized fashion.

We will denote as $\rho_{K}^{J M a x}$ the duty cycle which maximizes the sum dof, and as $\phi_{K}^{J M a x}$ the expected value of the sum dof achieved with JMax when the optimal duty cycle $\rho_{K}^{J M a x}$ is used. Although the exact analytical computation of $\rho_{K}^{J M a x}$ seems intractable, it is possible to make some assumptions to derive approximations of $\phi^{J \operatorname{Max}}$ and $\rho_{K}^{J \operatorname{Max}}$, which we will denote respectively as $\tilde{\phi}_{K}^{J M a x}$ and $\tilde{\rho}_{K}^{J M a x}$. Let us first consider a system with $K$ pairs and no coordination. We will denote as $p_{f}$ the probability that user $i$ gets no interference from user $j$. According to Eq. (4), $p_{f}=1-2 \rho$. If the transmit delays are random, the probability of one user having $n$ interferers is:

$$
\begin{aligned}
P_{n} & =\left(\begin{array}{c}
K-1 \\
n
\end{array}\right)\left(1-p_{f}\right)^{n} p_{f}^{K-1-n} \\
& =\left(\begin{array}{c}
K-1 \\
n
\end{array}\right)(2 \rho)^{n}(1-2 \rho)^{K-1-n},
\end{aligned}
$$

since there are $K-1$ potential interferers. Let us refer to the number of interferers of user $i$ as $n_{i}$, and let us order the users in ascending number of interferers so that $n_{1}<n_{2}<\ldots<n_{K}$. In order to simplify the analysis and arrive at an approximate result, we assume the dof of each pair to be mutually independent. Under this assumption, the $K-1$ free variables are used in order to remove interference from users, starting from the user with the fewest interferers, $n_{1}$, until all free variables are consumed. Therefore $l$ users are freed from interference, where $l$ is the biggest integer so that $\sum_{i=1}^{l} n_{i} \leq K-1$. Moreover we are left with $s<n_{l+1}$, $s \geq 0$ free variables, which are used to decrease the number of interferers of the $l+1$-th pair to $n_{l+1}-s$.

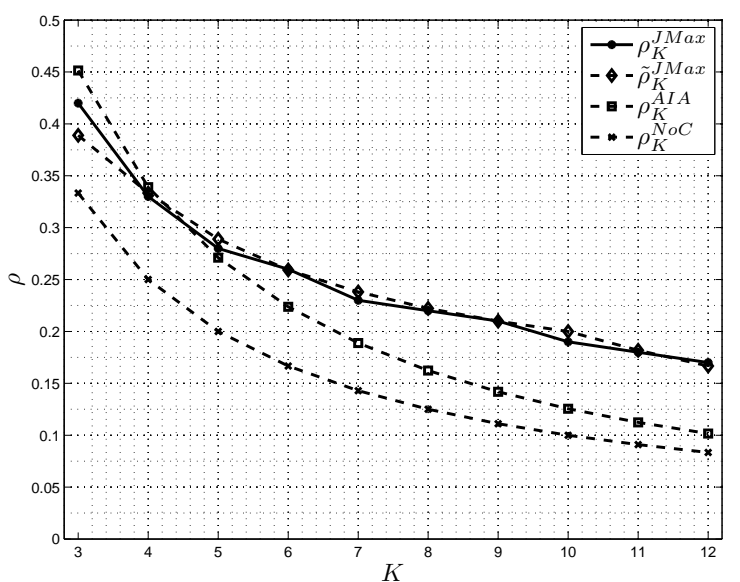

Fig. 7: Optimal duty cycle $\rho$ vs $K$. The solid line with round markers represents $\rho_{K}^{J M a x}$. The dashed line with diamond markers shows its approximation $\tilde{\rho}_{K}^{J M a x}$. The dashed lines with square and asterisk markers represent $\rho_{K}^{A I A}$ and $\rho_{K}^{N o C}$.

Using (14), the expression for $\tilde{\phi}_{K}^{J M a x}$ is the following:

$$
\tilde{\phi}_{K}^{J M a x}=l \rho+\rho(1-\rho)^{n_{l+1}-s}+\sum_{i=l+2}^{K} \rho(1-\rho)^{n_{i}} .
$$

Note that (25) is a polynomial on $\rho$ of degree smaller or equal than $K$. Hence, the duty cycle $\tilde{\rho}_{K}^{J M a x}$ which maximizes (25) can be obtained numerically in a very efficient manner using, for example, a gradient ascent algorithm.

The optimal duty cycle $\rho_{K}^{J M a x}$ and its approximation $\tilde{\rho}_{K}^{J M a x}$ as a function of $K$ are shown in Fig. 7. The figure shows how $\tilde{\rho}_{K}^{J M a x}$ is a very good approximation of $\rho_{K}^{J M a x}$. Moreover we can see how $\rho_{K}^{J M a x}$ and $\tilde{\rho}_{K}^{J M a x}$ are considerably larger than $\rho_{K}^{N o C}$ and $\rho_{K}^{A I A}$.

Fig. 8 shows $\phi_{K}^{J \operatorname{Max}}$ and its approximation $\tilde{\phi}_{K}^{J \operatorname{Max}}$ as a function of the number of pairs $K$. Both $\phi_{K}^{J M a x}$ and $\tilde{\phi}_{K}^{J M a x}$ increase with $K$, which was not the case for altruistic IA and the uncoordinated case where the dof decreased as $K$ increased. However, $\tilde{\phi}_{K}^{J M a x}$ is always smaller than $\phi_{K}^{J M a x}$, and the approximation is not as tight as it was for the duty cycle. It is quite remarkable that for $K \geq 5, \phi_{K}^{J M a x}>1$ and therefore, a system with 5 or more pairs using time IA and joint maximization provides on average more dof than an orthogonal resource allocation scheme. Moreover, our numerical analysis suggests that for increasing $K$ the probability of attaining more dof than an orthogonal scheme tends to 1 . For $K \geq 7$ the probability of achieving less than 1 sum dof is already smaller than $10^{-4}$. We conjecture from the plots that the sum dof grow without bound with $K$ even without bandwidth scaling, although sublinearly with the network size. 


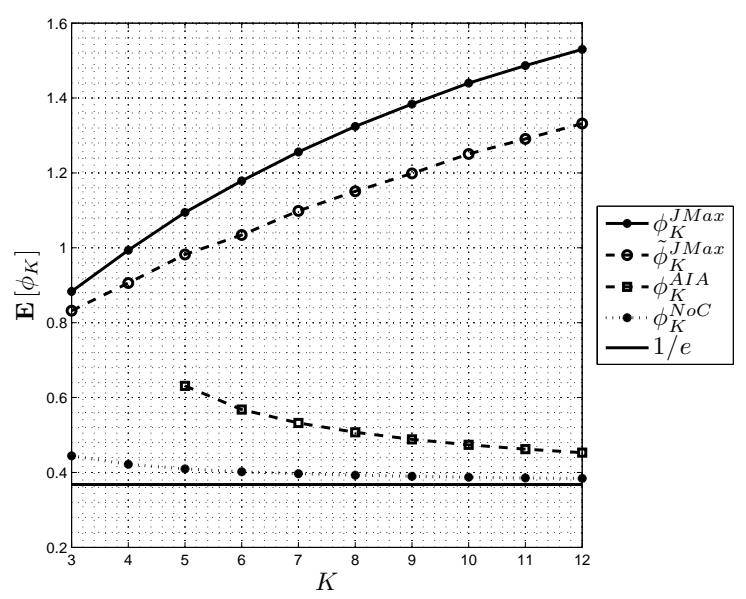

Fig. 8: Sum dof vs $K$ for the JMax scheme. The solid line with '*' markers shows $\phi_{K}^{J M a x}$, the dashed line with 'o' markers shows its approximation $\tilde{\phi}_{K}^{J M a x}$, the dashed line with square markers shows $\phi^{A I A}$, the dotted line with '*' markers shows $\phi^{N o C}$ and the solid line shows $\frac{1}{e}$.

The joint optimization problem does not scale well with the number of users $K$, since the number of variables to optimize is $K$ and therefore the dimension of the search space grows linearly with $K$. Hence, this scheme will not be practical for large values of $K$. However, for values of $K$ up to 10 the joint maximization is still practical and provides a gain in terms of dof. In this work the focus is on the achievability of dof rather than in developing low complexity algorithms to perform the joint maximization.

\section{APPLICATIONS FOR SATELLITE NETWORKS}

The results presented in Sections III and IV assume that the elements in the normalized propagation delay matrix $B$ are uniformly distributed between 0 and 1 . This assumption holds in reality only if the differences between the propagation delays $a_{i, j}$ are much larger than $T$. In this section we illustrate the applicability of time IA in satellite networks, which are characterized by long propagation delays and bandwidths in the order of MHz. We assume the $K$-user interference channel is formed by $K$ satellites which communicate with $K$ stations on ground, using the same frequency. Many reasons can be brought to operate multiple satellites in the same frequency, but two are of particular relevance. First of all, due to the high price of spectrum licenses, it is attractive to reuse the bandwidth very aggressively by deploying multiple satellites close to each other and using the same frequency. Moreover, it may be more viable from an economic point of view to first deploy one satellite and incrementally launch the rest of the constellation when the revenues pick up, rather than operating a single very large and very expensive satellite. Hence, a first investigation of how much capacity is achieved by time IA can have practical importance. Furthermore, we remark that transmit delay coordination is in fact already in place in some TDMA standards for the return link, like DVB-RCS [19], which require that the users' signals arrive at specific time instants at the satellite. These delay corrections are computed by the network gateway and therefore from a system point of view there would be almost no cost in implementing time IA for multiple satellites directed by a single gateway.

In our study, the focus is on geostationary satellites, because a large number of communication satellites are along this orbit. Moreover, the delay from a geostationary satellite to a point on ground changes very slowly over time, which simplifies the setup. We will assume that the $K$ satellites are equally spaced along the geostationary orbit so that they span between 24.5 and 25.5 degrees east, with a separation of $\frac{1}{K-1}^{\circ}$ between neighbor satellites. These orbital positions lie over Europe. The ground stations are randomly placed over Europe, being their latitude and longitude uniformly distributed in the ranges $\left[35^{\circ}, 55^{\circ}\right]$ north and $\left[-10^{\circ}, 20^{\circ}\right]$ east, respectively. We remark that only the positions of ground terminals are random and the satellite positions are fixed, whereas in the previous sections all nodes, transmitters and receivers, were randomly placed. In all cases the duty cycle is assumed to be $\tilde{\rho}_{K}^{J M a x}$, and the transmitters jointly coordinate to maximize the sum dof, which we will denote as $\phi_{K}^{\text {Sat }}$. In the results presented in this section the transmitter delays are numerically jointly optimized in order to maximize the sum dof $\phi_{K}^{\text {Sat }}$ according to the same method of Section IV-B.

Fig. 9 shows $\phi_{K}^{S a t}$ for the previously described satellite setting, as a function of $T$ for different values of $K$. The solid lines represent actual numerical results for the satellite scenario, whereas the dashed lines correspond to $\phi_{K}^{J M a x}$ under the assumption that the elements of the matrix $B$ are independent and uniformly distributed between 0 and 1 . In the figure we observe how for large values of $T, \phi_{K}^{S a t}<1$ (see Appendix $\mathrm{C}$ for a proof). For small values of $T \phi_{K}^{S a t} \approx \phi_{K}^{J M a x}$. In other words the sum dof in the satellite setting is very close the sum dof when the entries in $B$ are uniformly distributed from 0 and 1.

Let us define $\tilde{T}$ as the maximum value of $T$ for which $\phi_{K}^{S a t} / \phi_{K}^{J M a x} \geq 0.97$. In other words $\tilde{T}$ is the value of $T$ below which the elements of the matrix $B$ start to be sufficiently random. Fig. 10 depicts $\tilde{T}$ as a function of $K$. For example, for $K=3$ users, $T=500 \mu$ s is already enough to approach the performance of a random setting. However, as the number of users $K$ increases, $\tilde{T}$ decreases. For example, for $K=10$ users, a value of $T=56 \mu \mathrm{s}$ is required to approach the performance 
of the random setting. The necessity of a smaller $T$ is partly due to the smaller inter-satellite separation, as the whole constellation is constrained to be deployed in an orbital slot one degree wide.

In all the time IA schemes proposed in this work, inside each interval of length $T$ pair $i$ will have a continuous time $\alpha_{i} T$ in which the pair can communicate in the absence of interference. It is not necessary to fit one whole transmission frame inside $\alpha_{i} T$. However, in practical implementations many transmission symbols should fit in the interference free time $\alpha_{i} T$. Nevertheless, the symbol duration in satellite networks is generally very short. For example, in Ku-band the typical transponder bandwidth is $36 \mathrm{MHz}$. If the whole transponder is used to carry only one DVB-S2 channel [20], assuming a roll-off factor of 0.2 the baud rate is $30 \mathrm{Mbaud} / \mathrm{z}$, which translates into a symbol duration of $0.033 \mu \mathrm{s}$. This value is very small compared to the value of $T=56 \mu \mathrm{s}$ required for $K=10$. In higher frequency bands such as Ka-band channels can be even wider (in the order of $100 \mathrm{MHz}$ ) so the operation with $K=10$ would pose no challenge at all.

In the example provided, fixed terminals are assumed. However the position of the satellites in the geostationary orbit is not always constant, but changes with time. These changes are called orbit perturbations and make the satellite drift from its orbit. To counter act this drift satellites are equipped with rockets which are used to keep the satellite into the so called station-keeping box. For a geostationary satellite this box has a typical size of $\pm 0.05^{\circ}$ in latitude and longitude [21]. If we consider a satellite receiver placed in Munich, Germany, and a geostationary satellite at 25 degrees east, an excursion of 0.05 degrees in longitude changes the delay by 3.17 $\mu \mathrm{s}$. The drift of the satellite inside the station-keeping box is rather slow. It takes several hours for a satellite to reach the borders of its station-keeping box. Moreover, the satellite operator monitors continuously the position of the satellite and has a very accurate estimate of the satellite position at all times. Therefore the operator can compensate for the delay variation introduced by the drift. Hence, in the case of a geostationary satellite, after compensation for the delay variation a residual error several orders of magnitude lower than $3.17 \mu$ s would be left. Such an error would have a rather small effect in the sum dof.

It is possible to relax the constraints on $T$ by increasing the separation between satellites. This is not meaningful for stationary satellite terminals, since their antennas are very directive (their main lobe of the antenna is around $1^{\circ}$ wide). However, it is meaningful for mobile terminal, whose antennas must be small and have limited or no directivity. A multi-satellite constellation for mobile users has indeed been rolled out in the
XM-Sirius satellite radio system in order to provide satellite diversity [22], therefore such systems are in fact already reality and therefore the proposed scheme may be practically relevant.

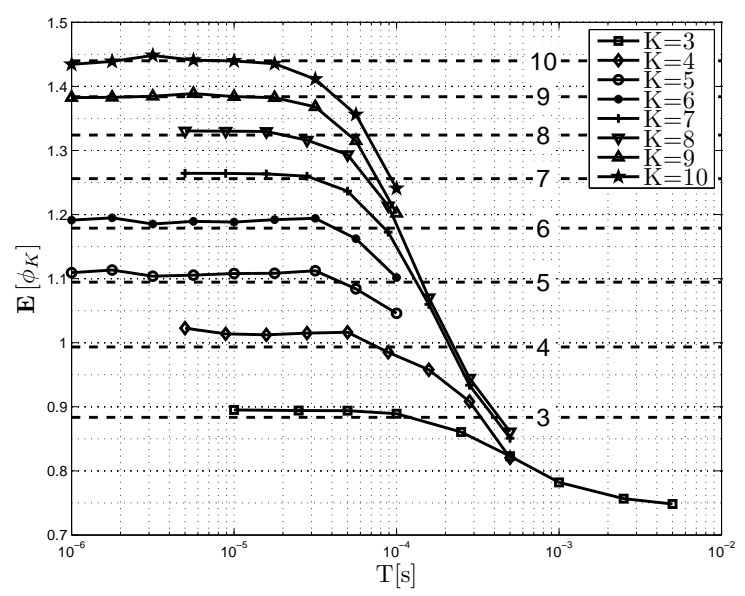

Fig. 9: $\phi_{K}^{S a t}$ for $\mathrm{K}$ ranging from 3 to 10 vs $T$ for the selected satellite scenario. The solid lines represent numerical results for Monte Carlo simulations in a satellite scenario, where markers represent the different values of $T$ used for the simulations. The dashed lines represent $\phi_{K}^{J M a x}$ for different values of $K$.

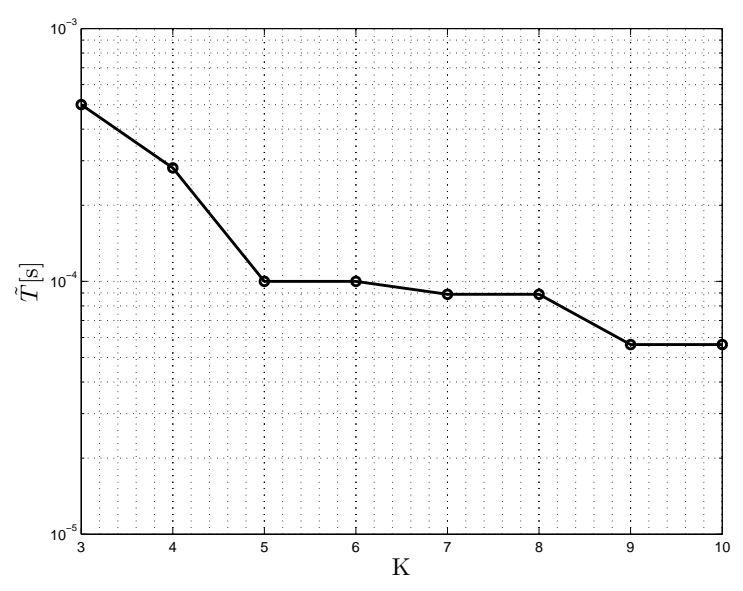

Fig. 10: $\tilde{T}$ vs $K$.

\section{Conclusions}

This work has studied the capacity improvements in terms of dof brought by time interference alignment in a more general setting than its predecessors [1], [13][15]. Three different time IA schemes have been studied. A scheme has been presented in which transmitters coordinate to jointly maximize the sum dof of a network. With such a scheme the sum dof grows as the number of pairs $K$ increases. The rise in the sum dof observed is less than linear in $K$, however, in contrast to some work 
in time IA [18], the schemes proposed in this work do not require bandwidth scaling (i.e., the increase of bandwidth with the number of pairs $K$ ). Furthermore for $K>5$ the proposed scheme provides on average more dof than an orthogonal scheme. It has also been shown how in the absence of coordination or in the case in which transmitters coordinate without taking into account the desired component at the receivers (altruistic IA), the sum dof of the network decreases as the number of pairs $K$ increases. Finally, the applicability of the scheme has been studied in a realistic multi-satellite network, and it has been shown how the proposed schemes could be practically relevant. In the present work, it has been assumed that all nodes have only one antenna. The more general case in which nodes have several antennas opens many new possibilities. For example, receivers with several antennas could employ zero forcing beamforming in order to reduce the number of interferers. The multiple antenna case has not been addressed in this work and is left for further study.

\section{ACKNOWLEDGMENT}

The authors would like to thank the Associate Editor and the Anonymous Reviewers for their comments that helped to significantly improve the paper. They would also like to thank M. Nikolaus (Universit at HamburgHarburg) for useful discussions.

\section{APPENDIX A}

DERIVATION OF THE PDF OF $\phi_{3}$

The pdf of the sum dof is obtained by convolution of the single user pdfs. For three users the expression obtained for $f\left(\phi_{3}\right)$ is the following:

$$
\begin{aligned}
f\left(\phi_{3}\right) & =a^{3} \delta\left(\phi_{3}\right)+3 a^{2} b \delta\left(\phi_{3}-\rho\right)+3 a b^{2} \delta\left(\phi_{3}-2 \rho\right) \\
& +b^{3} \delta\left(\phi_{3}-3 \rho\right)+p_{1}\left(\phi_{3}\right) \operatorname{rect}\left(\frac{\phi_{3}-\frac{\rho}{2}}{\rho}\right) \\
& +p_{2}\left(\phi_{3}\right) \operatorname{rect}\left(\frac{\phi_{3}-\frac{3 \rho}{2}}{\rho}\right)+p_{3}\left(\phi_{3}\right) \operatorname{rect}\left(\frac{\phi_{3}-\frac{5 \rho}{2}}{\rho}\right),
\end{aligned}
$$

where:

$$
\begin{aligned}
a & =\rho^{2} \\
b & =1-4 \rho+4 \rho^{2} \\
p_{1}\left(\phi_{3}\right) & =-6 p^{5}-6 p^{4} \phi_{3}+12 p^{4}+32 p^{3} \phi_{3}^{2}-48 p^{3} \phi_{3} \\
& +6 p^{2} \phi_{3}^{3}-48 p^{2} \phi_{3}^{2}+48 p^{2} \phi_{3}-9 p \phi_{3}^{4}+48 p \phi_{3}^{3} \\
& -48 p \phi_{3}^{2}-\frac{9}{5} \phi_{3}^{5}+18 \phi_{3}^{4}-48 \phi_{3}^{3}+32 \phi_{3}^{2} \\
p_{2}\left(\phi_{3}\right) & =\frac{303}{5} p^{5}+39 p^{4} \phi_{3}-198 p^{4}-118 p^{3} \phi_{3}^{2} \\
& 240 p^{3} \phi_{3}+78 p^{3}-12 p^{2} \phi_{3}^{3}+204 p^{2} \phi_{3}^{2} \\
& -426 p^{2} \phi_{3}+96 p^{2}++18 p \phi_{3}^{4}-96 p \phi_{3}^{3}+78 p \phi_{3}^{2} \\
& +96 p \phi_{3}-48 p+\frac{18}{5} \phi_{3}^{5}-36 \phi_{3}^{4}+114 \phi_{3}^{3} \\
& -136 \phi_{3}^{2}+48 \phi_{3} \\
p_{3}\left(\phi_{3}\right) & =-\frac{273}{5} p^{5}-33 p^{4} \phi_{3}+186 p^{4}+86 p^{3} \phi_{3}^{2} \\
& -192 p^{3} \phi_{3}+-42 p^{3}+6 p^{2} \phi_{3}^{3}-156 p^{2} \phi_{3}^{2} \\
& +378 p^{2} \phi_{3}-168 p^{2}-9 p \phi_{3}^{4}+48 p \phi_{3}^{3}-30 p \phi_{3}^{2} \\
& -96 p \phi_{3}+78 p-\frac{9}{5} \phi_{3}^{5}+18 \phi_{3}^{4}-66 \phi_{3}^{3}+104 \phi_{3}^{2} \\
& -66 \phi_{3}+12
\end{aligned}
$$

\section{APPENDIX B \\ MAXimization of $\mathbf{E}\left[\phi_{K}\right]$ FOR AIA}

According to (22), $\mathbf{E}\left[\phi_{K}\right]$ is a polynomial on $\rho$. Let us introduce the auxiliary variable $q=1-\rho . \mathbf{E}\left[\phi_{K}\right]$ will have the expression $\mathbf{E}\left[\phi_{K}\right]=\rho P(q)$, where $P(q)$ is a polynomial on $q$. The terms in $P(q)$ have degrees ranging from 1 to $(K-1)$. Let us assume that the term with the minimum degree in $P(q)$ has degree $b$, with $1<b<K-1$. It will also be assumed that $P(q)$ has a term of degree $c \geq b$. Now let us define a new polynomial $R(q)=P(q)+q^{b-1}-q^{b}-q^{c}+q^{c+1}$ with $k-$ $1>c>b>1$. $R(q)$ also complies with the constraints in (20) and (21). Let us define $z=c-b$, where $z \in \mathbb{N}$ and $z \geq 1$. We need to prove that $R(q)>P(q)$ :

$$
\begin{aligned}
R(q) & >P(q) \\
q^{b-1}-q^{b}-q^{c}+q^{c+1} & >0 \\
q^{b-1}\left(1-q-q^{c-b+1}+q^{c-b+2}\right) & >0 \\
q^{b-1}\left(1-q-q^{z+1}+q^{z+2}\right) & >0 \\
q^{b-1}\left(1-q-q^{z+1}(1-q)\right) & >0 \\
q^{b-1}\left((1-q)\left(1-q^{z+1}\right)\right) & >0 .
\end{aligned}
$$

Let us recall that $z \geq 1$ and $\frac{1}{2} \leq q<1 .{ }^{4}$ In this case $q^{b-1}>0,(1-q)>0$ and $\left(1-q^{z+1}\right)>0$. Therefore, the product of this three terms must also be greater than 0 . Hence, $\mathbf{E}\left[\phi_{K}\right]$ will be maximized when it has a term of degree one. In order to get a term of degree one, $K-2$ transmitters must set their transmit delays. A transmitter remains which can freely set its transmit delay to reduce by one the number of potential interferers at another receiver. Therefore $\mathbf{E}\left[\phi_{K}\right]$ is maximized for $u_{1}=1$, $u_{K-2}=1$ and $u_{K-1}=K-2$

\footnotetext{
${ }^{4}$ Let us remark that the assumption $0<\rho \leq \frac{1}{2}$ yields $\frac{1}{2} \leq q<1$.
} 


\section{APPENDIX C \\ $\phi_{K}^{S a t}$ WHEN ALL ELEMENTS OF $B$ TAKE THE SAME VALUE}

In this appendix we derive expressions for the sum dof when the transmit delays are jointly optimized in the special case in which all the elements in the normalized propagation delay matrix $B$ take the same value. In a real application scenario this is the case when the period of the time allocation $T$ is much bigger than the differences among the elements of $B$. This special case is equivalent to having only one receiver and $K$ transmitters in a TDMA manner.

Depending on the value of $\rho$ and $K$, a distinction among 3 different cases will be done:

1. $\rho \leq \frac{1}{K}$. In this case, it is possible to set the transmit delays $\Delta_{i}$ so that there is no mutual interference among pairs, as shown in Fig. 11a. The sum degrees of freedom will be $\phi_{K}^{S a t}=K \rho$

2. $\frac{1}{K}<\rho \leq \frac{1}{K-1}$. In this case, no matter how we set the transmit delays $\Delta_{i}$, there will be some interference among pairs, since it is equivalent to having a TDMA system in which the sum of the time allocation to the terminals exceeds $100 \%$, which leads to interference. However it is possible to set the transmit delays as shown in Fig. $11 \mathrm{~b}$ so that the mutual interference is minimized. Concretely, it is possible to set $\Delta_{i}$ so that the interference spans over a share $K \rho-1$ of the time. Therefore, the portion of time taken by the useful signals will be $\phi_{K}^{\text {Sat }}=1-(K \rho-1)=2-K \rho$.

3. $\rho>\frac{1}{K-1}$. This last case is similar to the previous one. Here, it is possible to set the transmit delays $\Delta_{i}$ as shown in Fig. 11c so that the interference spans over a share $\rho$ of time. The remaining share of the time, $1-\rho$ is free of interference. Therefore, $\phi_{K}^{S a t}=1-\rho$.

We remark that in all the cases $\phi_{K}^{\text {Sat }} \leq 1$. As we mentioned, when all elements in $B$ take exactly the same value, the system is equivalent to a TDMA setting with $K$ transmitters and 1 single receiver. Obviously the maximum sum dof achievable in this case is 1 and corresponds to the case in which $\rho K=1$.

\section{REFERENCES}

[1] V. R. Cadambe and S. A. Jafar, "Interference alignment and spatial degrees of freedom for the K user interference channel," IEEE Trans. Inform. Theory, vol. 54, no. 8, pp. 3425-3441, Aug. 2008.

[2] _ - "Interference alignment and the degrees of freedom of wireless X networks," IEEE Trans. Inform. Theory, vol. 55, no. 9, pp. 3893-3908, Sep. 2009.

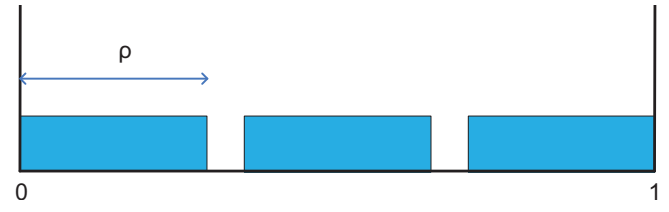

(a) $\rho \leq \frac{1}{K}$

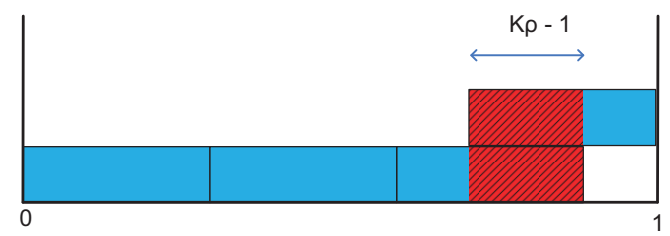

(b) $\frac{1}{K}<\rho \leq \frac{1}{K-1}$

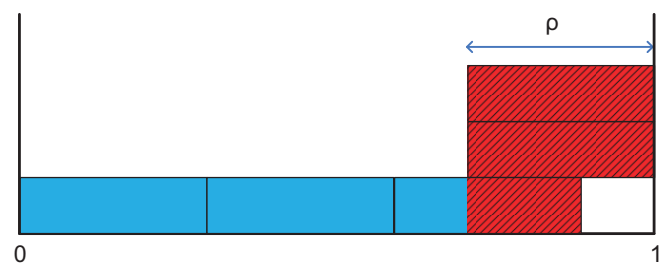

(c) $\rho>\frac{1}{K-1}$

Fig. 11: Received signal at the $K$ receivers, when all elements in $B$ have the same value. The blocks represent the transmission from different terminals. Solid blocks represent non interfered transmissions, and striped blocks represent the share of the transmission which gets interfered and, therefore, does not contribute to the sum dof. It has been assumed that the time slot duration is $T=1$.

[3] C. M. Yetis, T. Gou, S. A. Jafar, and A. H. Kayran, "On feasibility of interference alignment in MIMO interference networks," IEEE Trans. Signal Processing, vol. 58, no. 9, pp. 4771-4782, Sep. 2010.

[4] K. Gomadan, V. R. Cadambe, and S. A. Jafar, "Approaching the capacity of wireless networks through distributed interference alignment," in Proc. 2008 IEEE Global Telecommun. Conf., New Orleans, Lousiana, USA, Dec. 2008.

[5] O. El Ayach, S. Peters, and R. Heath, "The feasibility of interference alignment over measured MIMO-OFDM channels," IEEE Trans. Veh. Technol., vol. 59, no. 9, pp. 4309-4321, Nov. 2010.

[6] M. Maddah-Ali, A. Motahari, and A. Khandani, "Signaling over mimo multi-base systems: Combination of multi-access and broadcast schemes," in Proc. 2006 IEEE Int. Symposium on Inform. Theory, Seattle, Washington, USA, Jul. 2006, pp. 2104 2108.

[7] D. Schmidt, W. Utschick, and M. Honig, "Large System Performance of Interference Alignment in Single-Beam MIMO Networks," in Proc. 2010 IEEE Global Telecommun. Conf., Miami, Florida, USA, Dec. 2010. 
[8] C. M. Yetis, S. A. Jafar, and A. H. Kayran, "Feasibility conditions for interference alignment," in Proc. 2009 IEEE Global Telecommun. Conf., Honolulu, Hawaii, USA, Dec. 2009.

[9] V. R. Cadambe, S. A. Jafar, and S. Shamai, "Interference alignment on the deterministic channel and application to fully connected gaussian interference networks," IEEE Trans. Inform. Theory, vol. 55, pp. 269-274, Jan. 2009.

[10] S. Peters and R. W. Heath Jr., "Interference alignment via alternating minimization," in Proc. 2009 IEEE Int. Conf. on Acoustics, Speech and Signal Processing, Taipei, Taiwan, Oct. 2009, pp. 2445-2448.

[11] D. A. Schmidt, C. Shi, R. A. Berry, M. L. Honig, and W. Utschick, "Minimum mean squared error interference alignment," in Asilomar Conf. on Signals, Systems and Computers, Asilomar, California, USA, Nov. 2009, pp. 1106-1110.

[12] S. Gollakota, S. D. Perli, and D. Katabi, "Interference alignment and cancellation," in ACM SIGCOMM, Barcelona, Spain, Aug. 2009, pp. 159-170.

[13] V. Cadambe and S. Jafar, "Degrees of freedom of wireless networks - what a difference delay makes," in Asilomar Conf. on Signals, Systems and Computers, Asilomar, California, USA, Nov. 2007, pp. 133-137.

[14] R. Mathar and G. Bocherer, "On spatial patterns of transmitterreceiver pairs that allow for interference alignment by delay," in Proc. of the 2009 Int. Conf. on Signal Processing and Commun. Systems, Omaha, Nebraska, USA, Sep. 2009.

[15] R. Mathar and M. Zivkovic, "How to position n transmitterreceiver pairs in n-1 dimensions such that each can use half of the channel with zero interference from the others," in Proc. 2009 IEEE Global Telecommun. Conf., Honolulu, Hawaii, USA, Dec. 2009.

[16] H. Maier, J. Schmitz, and R. Mathar, "Cyclic interference alignment by propagation delay," in Proc. 50th Allerton Conf. on Commun., Control, and Computing, Monticello, Illinois, USA, Oct. 2012, pp. 1761-1768.

[17] M. Torbatian and M. Damen, "Asynchronous interference alignment," in Proc. 48th Allerton Conf. on Commun., Control, and Computing, Monticello, Illinois, USA, Oct. 2010, pp. 262-269.

[18] L. Grokop, D. Tse, and R. Yates, "Interference alignment for lineof-sight channels," IEEE Trans. Inform. Theory, vol. 57, no. 9, pp. 5820-5839, Sep. 2011.

[19] ETSI EN 301790 v1.5.1; Digital Video Broadcasting (DVB); Interaction channel for satellite distribution systems, European Telecommunications Standards Institute (ETSI) Std., 2009.

[20] ETSI EN 302307 v1.3.1; Digital Video Broadcasting (DVB); Second generation framing structure, channel coding and modulation systems for Broadcasting, Interactive Services, News Gathering and other broadband satellite applications (DVB-S2), European Telecommunications Standards Institute (ETSI) Std., 2013.

[21] G. Maral, M. Bousquet, and Z. Sun, Satellite Communications Systems: Systems, Techniques and Technology. Chichester, UK: Wiley, 2009.

[22] S. DiPierro, R. Akturan, and R. Michalski, "Sirius XM Satellite Radio system overview and services," in Proc. 2010 Advanced
Sat. Multimedia Systems Conf. (ASMS) and Signal Processing for Space Commun. Workshop (SPSC), Cagliari, Italy, Sep. 2010, pp. 506-511.

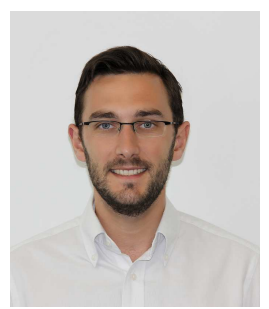

Francisco Lázaro Blasco was born in Zaragoza, Spain, in 1983. He received his Telecommunication Engineering degree from the Universidad de Zaragoza, Spain, in 2006. In 2008 he joined the Institute for Communications and Navigation at the German Aerospace Center (DLR), as a research scientist, where he is working toward his $\mathrm{PhD}$. His main research interests are rate-less codes and multi-user communications with focus on satellite and space communications. During the past years he has been involved in many national and international projects. He is an IEEE student member and servers as IEEE reviewer for Journals and Conferences.

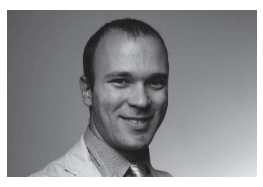

Francesco Rossetto (S'06, M'09) received the "laurea" (equivalent to MS) and the $\mathrm{PhD}$ in Telecommunications Engineering in 2005 and 2009, respectively, from the University of Padova, Padova, Italy. In 2008 he was on leave at the University of California, San Diego, working for the MURI project. Between 2009 and 2012 he has been with the DLR (German Aerospace Center) in Munich, Germany, for the investigation of multiple access strategies in satellite networks. Since 2012 he is with the Mobile Communications division of Rohde \& Schwarz, developing mobile communication testers. Between 2011 and 2013 he had a teaching appointment at the Munich Technical University. His research interests include multiuser communication, network coding and cross layer design. His corporate experience includes a summer internship in 2006 at the Ericsson Eurolabs, in Aachen, Germany. 


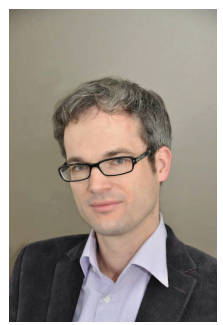

Gerhard Bauch received the Dipl.-Ing. and Dr.-Ing. degree in Electrical Engineering from Munich University of Technology (TUM) in 1995 and 2001, respectively, and the Diplom-Volkswirt (master in economics) degree from FernUniversitaet Hagen in 2001. In 1996, he was with the German Aerospace Center (DLR), Oberpfaffenhofen, Germany. From 1996-2001 he was member of scientific staff at Munich University of Technology (TUM). In 1998 and 1999 he was visiting researcher at AT\&T Labs Research, Florham Park, NJ, USA. In 2002 he joined DOCOMO Euro-Labs, Munich, Germany, where he has been managing the Advanced Radio Transmission Group. In 2007 he was additionally appointed Research Fellow of DOCOMO Euro-Labs. From 2003-2008 he was an adjunct professor at Munich University of Technology. In 2007 he was a visiting professor teaching courses at the University of Udine in Italy and at the Alpen-Adria-University Klagenfurt in Austria. He was a full professor at the Universität der Bundeswehr Munich from 2009-2012. Since October 2012 he is head of the Institute of Communications at Hamburg University of Technology. He received best paper awards of the European Personal Mobile Communications Conference (EPMCC) 1997, Globecom 2008, Globecom 2009 and the IEEE International Conference on Communications (ICC)) 2009, the Texas Instruments Award of TUM 2001, the award of the German Information Technology Society (ITG in VDE) 2002 (ITG Foerderpreis) and the literature award of the German Information Technology Society (ITG in VDE) 2007 (ITG-Preis). $\mathrm{He}$ was appointed as distinguished lecturer of the IEEE Vehicular Technology Society 2011-2015. He is a Senior Member of the IEEE and serves as the president of the Communications Society German chapter since 2013. He is also a member of the German Information Technology Society (ITG in VDE (Association for Electrical, Electronic \& Information Technologies)) where he serves as a member of the committee "Information and System Theory". He has (co-)authored a textbook on "Contemporary Communications Systems" as well as more than 150 scientific papers in major journals and international conferences. 